EESTI NSV TEADUSTE AKADEEMIA TOIMETISED, 29. KOIDE

FOOSIKA * MATEMAATIKA. 1980, NR. 2

ИЗВЕСТИЯ АКАДЕМИИ НАУК ЭСТОНСКОИ ССР. ТОМ 29 ФИЗИКА * МАТЕМАТИКА, 1980, № 2

удК 658.012

Ю. ТАММЕЛА

\title{
ОПТИМАЛЬНОЕ УПРАВЛЕНИЕ ПРОИЗВОДСТВЕННЫМИ ПРОЦЕССАМИ СО СЛОЖНОЙ СТРУКТУРОЙ. МОДЕЛЬ С ПОЛНОЙ ИНФОРМАЦИЕЙ
}

\author{
(Представил Н. Алумяз)
}

\section{1. Введение}

Стохастический производственный процесс обычно определяется как простая последовательность технологических операций $\left[{ }^{1-3}\right]$ и описывается математической моделью как некоторый частично наблюдаемый марковский процесс с конечным временем планирования. Таков и процесс, описанный в $\left[{ }^{4}\right]$, но уже с более сложной структурой, которая представлена ориентированным графом без контуров. В [5] анализировались процессы с бесконечным временем планирования и структура их задавалась ориентированным графом с контурами (петлями) длиной единица. Наличие контуров в технологической схеме означает, что объект может быть подвергнут обработке неограниченное число раз. В таких случаях стратегию управления приходится задавать в виде функции от истории сколь угодно большой длины. Однако построение стратегии значительно упрощается, если предположить, что состояние объекта полностью наблюдаемо на всех стадиях его обработки.

В настоящей работе изучаются именно такие, полностью наблюдаемые, процессы со сложной структурой, причем, на структуру никаких ограничений не накладывается. Дается формальное определение элементов модели. Предполагается, что с каждым изменением состояния объекта связана плата за его обработку (и контроль) - текущая плата. По окончании процесса обработки получается доход от продажи изделия - финальный доход. Процесс обработки объекта следует проводить так, чтобы математическое ожидание разности финального дохода и суммарной текущей платы достигало максимума. Поставленная таким образом задача описывается более формально, т. е. она сводится к задаче стохастического динамического программирования $\left[{ }^{6}\right]$ или, что то же самое, к задаче управления марковским процессом $\left[{ }^{7-9}\right]$. Оптимальная стратегия управления таким процессом (на примере процесса фотолитографии) строится при помощи итеративного алгоритма Ховарда $\left[{ }^{8,9}\right]$.

\section{2. Пример процесса со сложной структурой и элементы его модели}

В качестве примера стохастического производственного процесса со сложной структурой рассмотрим один из этапов изготовления силовых полупроводниковых приборов - процесс фотолитографии. На рисунке структура этого процесса представлена в виде ориентированного графа, 


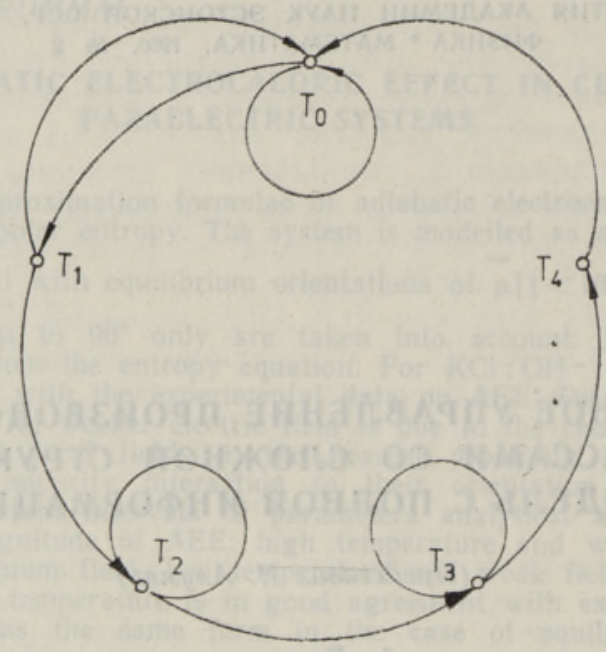

Структура процесса фотолитографии.

вершинам которого соответствуют контрольные операции, а дугам операции обработки.

Исходным объектом труда для фотолитографической обработки является соответствующим образом подготовленный диск кремния. На диск наносится слой фоточувствительного материала - фоторезиста. Диск с фоторезистом поступает на операцию контроля качества (вершина $T_{0}$ ). Если качество фоторезиста отвечает установленным требованиям, т. е. если объект признается годным для дальнейшей обработки, то диск передается на операцию экспонирования и проявления (дуга $\left.\left(T_{0}, T_{1}\right)\right)$. На поверхности диска получается фоторисунок, качество которого проверяется на следующей контрольной операции (вершина $T_{1}$ ). Диск с годным фоторисунком поступает на операцию травления (дуга $\left.\left(T_{1}, T_{2}\right)\right)$, затем на операцию контроля (вершина $\left.T_{2}\right)$ и т. д. Если же на контрольной операции $T_{n}, n=0,1, \ldots, 4$, объект признается негодным, он либо выбраковывается (процесс изготовления полупроводникового прибора из данного диска кремния прекращается), либо поступает на исправление (проводится некоторая дополнительная обработка диска на операции $\left.\left(T_{n}, T_{m}\right), m \leqslant n\right)$. Для процесса литографии основным исправлением (операцией типа $\left(T_{n}, T_{0}\right)$ ) является регенерация объекта, т. е. снятие с поверхности диска кремния нанесенного в процессе литографии слоя. Процесс фотолитографии (над данным объектом) длится до тех пор, пока объект не достигнет конечной стадии обработки, т. е. пока он не будет признан годным на контрольной операции $T_{4}$ или не будет выбракован.

Обозначим множество контрольных операций через $T^{\prime}$, множество операций обработки через $\Delta^{\prime}$ и предположим, что имеет место включение $\Delta^{\prime} \subset T^{\prime} \times T^{\prime}$. Будем говорить, что изучаемый процесс имеет сложную структуру, если граф $\left(T^{\prime}, \Delta^{\prime}\right)$ содержит контуры.

Предполагая, что состояние объекта наблюдаемо на всех стадиях обработки, мы ввели тем самым описание контрольных операций процесса. Для описания операций обработки естественно ввести набор отображений $\left(F_{t}: X \times Y \times \Theta \rightarrow X, t \in \Delta^{\prime}\right)$, где $X-$ пространство состояний объекта, $Y$ - множество способов его обработки и $\Theta-$ множество значений случайного фактора. Смысл этих отображений состоит в следующем. Если $n$-й операцией обработки объекта является операция $t$, 
которая проводится способом $y$, то состояние объекта $x_{n}$ после обработки дается выражением

$$
x_{n}=F_{t}\left(x_{n-1}, y, \theta\right),
$$

где $\theta-$ значение случайного фактора.

Случайный фактор может быть задан набором $\left(\pi_{t_{y}}: t \in \Delta^{\prime}, y \in Y\right)$ распределений вероятностей на множестве $\Theta$. Заметим, что отображение $F_{t}$ и набор распределений $\left(\pi_{t_{y}}: y \in Y\right)$ при помощи выражения

$$
p^{\prime}\left(x^{\prime \prime} \mid x^{\prime}, t, y\right)=\pi_{t_{y}}\left\{\theta: F_{t}\left(x^{\prime}, y, \theta\right)=x^{\prime \prime}\right\}, \quad x^{\prime \prime} \in X,
$$

определяют на множестве $X$ распределение вероятностей $p^{\prime}$. Именно это, зависящее от параметров $x \in X, t \in \Delta^{\prime}$ и $y \in Y$, распределение вероятностей $p^{\prime}(\cdot \mid x, t, y)$ на множестве $X$ мы и называем моделью операций обработки.

Процесс изготовления изделия начинается с контроля его исходного состояния $x_{0}$ на одной из контрольных операций $\tau_{0}$. По результатам контроля выбирается как первая операция обработки $t_{1}$, так и способ ее проведения $y_{1}$. Допустимы при этом лишь операции типа $\left(\tau_{0}, \tau\right)$. После операции $t_{1}=\left(\tau_{0}, \tau_{1}\right)$ объект переходит в новое состояние $x_{1}$, которое наблюдается на контрольной операции $\tau_{1}$. По результатам наблюдения выбирается новая операция обработки $t_{2}=\left(\tau_{1}, \tau_{2}\right)$ и т. д.

Обозначим множество всех неотрицательных целых чисел через $N_{0}$ и предположим, что процесс обработки объекта был остановлен после проведения $v$ операций $\left(v \in N_{0}\right)$. В этом случае история процесса может быть задана последовательностью

$$
l_{v}=x_{0} t_{1} y_{1} x_{1} \ldots t_{v} y_{v} x_{v}=x_{0} \tau_{0} \tau_{1} y_{1} x_{1} \ldots \tau_{v-1} \tau_{v} y_{v} x_{v}
$$

Определим величины, необходимые для оценивания всех возможных историй. Предположим, что за обработку объекта на операции $t$ способом $y$ и за контроль после обработки приходится платить $c(x, t, y)$ единиц $(x-$ состояние объекта до обработки). Неотрицательную функцию $c$, при помощи которой задаются величины $c(x, t, y), x \in X, t \in \Delta^{\prime}$, $y \in Y$, назовем текущей платой.

Конечное состояние объекта $x_{v}$ является одним из факторов, определяющих доход $h$ от использования изделия. Допустим, что этот доход $h$ зависит еще и от того, на какой из контрольных операций $\tau_{v} \in T^{\prime}$ было принято решение остановить процесс обработки, и от того, каким было решение $y_{v+1} \in Y$ относительно способа использования изделия. Действительную функцию $h$, которая определена на множестве $X \times T^{\prime} \times Y$, назовем, соответственно, финальным доходом. Тогда при оптимальном использовании изделия получается доход

$$
h\left(x_{v}, \tau_{v}\right)=\max _{y_{v+1} \in Y}\left\{h\left(x_{v}, \tau_{v}, y_{v+1}\right)\right\}
$$

и оценкой истории $l_{v}$ является величина

$$
R_{v}\left(l_{v}\right)=h\left(x_{v}, \tau_{v}\right)-\sum_{n=1}^{v} c\left(x_{n-1}, t_{n}, y_{n}\right) .
$$

Наша цель - найти закон управления производственным процессом, при котором оценка процесса (математическое ожидание величины $\left.R_{v}\left(l_{v}\right), v \in N_{0}\right)$ достигнет максимума. 


\section{3. Математическая формулировка задачи управления}

Задачу управления стохастическим производственным процессом переформулируем в терминах теории управляемых марковских процессов. Модель управляемого марковского процесса есть $\left[{ }^{6,7}\right]$ совокупность $Z=(S, A, D, p, r)$. Определим эту совокупность при помощи следующих выражении:

- пространство состояний

$$
S=X \times T
$$

где множество $T=T^{\prime} \cup\left\{T_{N}\right\}, T_{N} \equiv T^{\prime}\left(T_{N}\right.$ - конечная стадия процесса; $N$ - количество контрольных операций);

- пространство управлений

$$
A=T \times Y
$$

- множества допустимых управлений

$D\left(s_{n}\right)=D\left(x_{n}, \tau_{n}\right)=\left\{(\tau, y):\left(\tau_{n}, \tau\right) \in \Delta^{\prime} U\left\{\left(\tau_{n}, T_{N}\right)\right\}, y \in Y\right\}, \quad s_{n} \in S ;$

- распределение вероятностей $p\left(\cdot \mid s_{n}, a_{n+1}\right)$ на множестве $S$ зависит от параметров $s_{n} \in S$ и $a_{n+1} \in D\left(s_{n}\right)$ и удовлетворяет выражению

$$
\begin{gathered}
p\left(s \mid s_{n}, a_{n+1}\right)=p\left(x, \tau \mid x_{n}, \tau_{n}, \tau_{n+1}, y_{n+1}\right)= \\
=\delta\left(\tau_{n+1} \mid T_{N}\right) \delta\left(x \mid x_{n}\right) \delta\left(\tau \mid \tau_{n+1}\right)+ \\
+\left(1-\delta\left(\tau_{n+1} \mid T_{N}\right)\right) p^{\prime}\left(x \mid x_{n}, \tau_{n}, \tau_{n+1}, y_{n+1}\right) \delta\left(\tau \mid \tau_{n+1}\right),
\end{gathered}
$$

где $s \in S$ и $\delta(\cdot \mid \cdot)$ - символ Кронекера *;

- функция доходов $r$ удовлетворяет выражению

$$
r\left(s_{n}, a_{n+1}\right)=r\left(x_{n}, \tau_{n}, \tau_{n+1}, y_{n+1}\right)=\left(1-\delta\left(\tau_{n} \mid T_{N}\right)\right) \times
$$

$\times\left[\delta\left(\tau_{n+1} \mid T_{N}\right) h\left(x_{n}, \tau_{n}, y_{n+1}\right)-\left(1-\delta\left(\tau_{n+1} \mid T_{N}\right)\right) c\left(x_{n}, \tau_{n}, \tau_{n+1}, y_{n+1}\right)\right]$,

где $\left(s_{n}, a_{n+1}\right) \in\{(s, a): s \in S$ и $a \in D(s)\}$.

Задачу построения оптимальной стратегии управления марковским процессом (1)-(5) будем называть задачей управления производственным процессом со структурой $\left(T^{\prime}, \Delta^{\prime}\right)$.

Стратегией управления стационарным марковским процессом называется $\left[{ }^{6-9}\right]$ отображение $f: S \rightarrow A$ со свойством $f(s) \in D(s)$. Начальное состояние $s_{0}=\left(x_{0}, \tau_{0}\right)$, переходная функция $p\left(\cdot \mid s_{n}, a_{n+1}\right)$ и стратегия $f$ определяют распределение вероятностей $P$ в пространстве всех историй процесса: вероятность $\mathbf{P}_{s_{0}}{ }^{f}\left(l_{v}\right)$ истории $l_{v}=s_{0} a_{1} s_{1} \ldots a_{v} s_{v} \in L_{v}=$ $=S \times A \times S \times \ldots \times A \times S$ при стратегии $f$ определяется выражением

$$
\mathbf{P}_{s_{0}}^{f}\left(l_{v}\right)=\prod_{n=1}^{v} p\left(s_{n} \mid s_{n-1}, a_{n}\right) \delta\left(a_{n} \mid f\left(s_{n-1}\right)\right) .
$$

Величина

$$
\omega(f)=\sum_{v \in N_{0}} \sum_{l_{v} \in L_{v}} \mathbf{P}_{s_{0}}^{f}\left(l_{v}\right) r\left(s_{v}, a_{v+1}\right)
$$

(ожидаемый суммарный доход) называется оценкой стратегии $f$. Верх-

*. $\delta(x \mid y)=\left\{\begin{array}{l}1, \text { если } x=y, \\ 0 \text { в остальных случаях. }\end{array}\right.$ 
няя грань $v$ функции $\omega(f)$ называется оценкой процесса. Стратегия $f$ является оптимальной, если ее оценка $\omega(f)=v$. Методы построения оптимальной стратегии изложены, например, в $\left[{ }^{8,9}\right]$.

\section{4. Построение оптимальной стратегии}

Предположим, что операции процесса фотолитографии не управляемы, т. е. существует один единственный способ их проведения. Обрабатываемый объект - диск кремния - опишем с точностью «годен-негоден», т. е. положим $X=\{0,1\}$, где $X=0$ обозначает состояние «годен», и $X=1$ - состояние «негоден». Модель основных операций $t=$ $=\left(T_{n-1}, T_{n}\right), 0<n<5=N$, зададим при помощи матрицы

$$
P^{\prime}=\left(p_{i j}^{\prime}\right)=\left(p^{\prime}(j-1 \mid i-1, t, y)\right)=\left(\begin{array}{ccc}
\varrho & 1-\varrho \\
0 & 1
\end{array}\right),
$$

а модель операции исправления $t=\left(T_{m}, T_{n}\right) \in \Delta^{\prime}, m \geqslant n$, - при помощи матрицы

$$
P^{\prime \prime}=\left(p_{i j}^{\prime \prime}\right)=\left(p^{\prime}(j-1 \mid i-1, t, y)\right)=\left(\begin{array}{cc}
\varrho & 1-\varrho \\
\varrho & 1-\varrho
\end{array}\right) .
$$

Положим, что параметр $\mathrm{Q}=0,9$, текущая плата $c(x, t, y) \equiv 1$ и финальный доход

$$
h(x, \tau, y)=\left\{\begin{array}{cl}
h=5, \text { если } x=0 \text { и } \tau=T_{4} ; \\
0 \quad \text { в остальных случаях. }
\end{array}\right.
$$

Введенные таким образом элементы модели процесса фотолитографии совместно с его структурой (рисунок) задают задачу управления процессом. Решим эту задачу при помощи итеративного алгоритма Ховарда $\left[{ }^{8,9}\right]$.

В качестве исходной выбираем стратегию, которая предпишет выбраковку негодного объекта на всех стадиях обработки, а годный объект направит на следующую основную операцию. Другими словами, выбираем исходное отображение $f: X \times T \rightarrow T \times Y$ такое, что $f\left(0, T_{n-1}\right)=$ $=\left(T_{n}, y\right)$ и $f\left(1, T_{n-1}\right)=\left(T_{5}, y\right)$ (см. первые строки в таблице).

Итерационный алгоритм Ховарда состоит из процедуры определения весов и процедуры улучшения решения. При определении весов решается система уравнений

$$
v\left(s^{\prime}\right)=q\left(s^{\prime} ; f\right)+\sum_{s^{\prime \prime} \in S} p\left(s^{\prime \prime} \mid s^{\prime}, f\left(s^{\prime}\right)\right) v\left(s^{\prime \prime}\right), \quad s^{\prime} \in S,
$$

где непосредственно получаемый доход

$$
q\left(s^{\prime} ; f\right)=\sum_{s^{\prime \prime} \in S} p\left(s^{\prime \prime} \mid s^{\prime}, f\left(s^{\prime}\right)\right) r\left(s^{\prime}, f\left(s^{\prime}\right)\right) .
$$

При улучшении решения приближенные оценки $v(s), s \in S$, используются для определения критерия управления

$$
q(s ; a(s))+\sum_{s^{\prime} \in S} p\left(s^{\prime} \mid s, a(s)\right) v\left(s^{\prime}\right) .
$$

В соответствии с этим критерием получаем набор управлений $(a(s)$ : $: s \in S)$, который принимаем за новую стратегию и переходим к определению весов.

Результаты проведенных по алгоритму Ховарда расчетов даны в 
таблице. На третьем итерационном цикле $(i=3)$ исходная и улучшенная стратегии совпадают. Оптимальность этой стратегии можно перепроверить путем подстановки найденных на третьей итерации оценок $v(x, \tau), x \in X, \tau \in T$ в уравнение оптимальности

$$
v\left(s^{\prime}\right)=\sup _{a \in D(s)}\left\{r\left(s^{\prime}, a\right)+\sum_{s^{\prime \prime} \in S} p\left(s^{\prime \prime} \mid s^{\prime}, a\right) v\left(s^{\prime \prime}\right)\right\},
$$

откуда, учитывая ранее приведенные определения (1)-(5), получим, что при всех $x \in X$ и $\tau \in T^{\prime}$ оценка $v(x, \tau)$ должна удовлетворять уравнению

$$
\begin{gathered}
v(x, \tau)=\max \left\{\max _{y \in Y}\{h(x, \tau, y)\}, \max _{\left(\tau, \tau^{\prime}, y\right) \in \Delta^{\prime} \times Y}\left\{\sum_{x \in X} p^{\prime}\left(x^{\prime} \mid x, \tau, \tau^{\prime}, y\right) v\left(x^{\prime}, \tau^{\prime}\right)-\right.\right. \\
\left.\left.-c\left(x, \tau, \tau^{\prime}, y\right)\right\}\right\} .
\end{gathered}
$$

Легко проверить, что найденные на третьей итерации оценки удовлет-

\begin{tabular}{|c|c|c|c|c|c|c|c|}
\hline \multirow{2}{*}{$\begin{array}{c}\text { Но- } \\
\text { мер } \\
\text { цикла, } \\
i\end{array}$} & \multirow{2}{*}{$\begin{array}{c}\text { Рассчитываемая } \\
\text { величина }\end{array}$} & \multicolumn{6}{|c|}{ Стадия обработки $T_{n}$} \\
\hline & & $n=0$ & $n=1$ & $n=2$ & $n=3$ & $n=4$ & $n=5$ \\
\hline 1 & $\begin{array}{l}f\left(0, T_{n}\right) \\
f\left(1, T_{n}\right) \\
q\left(0, T_{n} ; f\right) \\
q\left(1, T_{n} ; f\right) \\
v\left(0, T_{n}\right) \\
v\left(1, T_{n}\right)\end{array}$ & $\begin{array}{c}T_{1}, y \\
T_{5}, y \\
-1 \\
0 \\
-0,158 \\
0\end{array}$ & $\begin{array}{l}T_{2}, y \\
T_{5,}, y \\
-1 \\
0 \\
0,935 \\
0\end{array}$ & $\begin{array}{l}T_{3}, y \\
T_{5}, y \\
-1 \\
0 \\
2,15 \\
0\end{array}$ & $\begin{array}{c}T_{4}, y \\
T_{5}, y \\
-1 \\
0 \\
3,5 \\
0\end{array}$ & $\begin{array}{c}T_{5}, y \\
T_{5}, y \\
5 \\
0 \\
5 \\
0\end{array}$ & $\begin{array}{c}T_{5,}, y \\
T_{5}, y \\
0 \\
0 \\
0 \\
0\end{array}$ \\
\hline 2 & $\begin{array}{l}f\left(0, T_{n}\right) \\
f\left(1, T_{n}\right) \\
q\left(0, T_{n} ; f\right) \\
q\left(1, T_{n} ; f\right) \\
v\left(0, T_{n}\right) \\
v\left(1, T_{n}\right)\end{array}$ & $\begin{array}{c}T_{5 .} y \\
T_{5 . y} y \\
0 \\
0 \\
0 \\
0\end{array}$ & $\begin{array}{l}T_{2, y} \\
T_{5}, y \\
-1 \\
0 \\
1,277 \\
0\end{array}$ & $\begin{array}{l}T_{3}, y \\
T_{2}, y \\
-1 \\
-1 \\
2,388 \\
1,277\end{array}$ & $\begin{array}{l}T_{4}, y \\
T_{3}, y \\
-1 \\
-1 \\
3,5 \\
2,388\end{array}$ & $\begin{array}{c}T_{5}, y \\
T_{5}, y \\
5 \\
0 \\
5 \\
0\end{array}$ & $\begin{array}{c}T_{5}, y \\
T_{5}, y \\
0 \\
0 \\
0 \\
0\end{array}$ \\
\hline 3 & $\begin{array}{l}f\left(0, T_{n}\right) \\
f\left(1, T_{n}\right) \\
q\left(0, T_{n} ; f\right) \\
q\left(1, T_{n} ; f\right) \\
v\left(0, T_{n} ; f\right) \\
v\left(1, T_{n} ; f\right)\end{array}$ & $\begin{array}{l}T_{1}, y \\
T_{5}, y \\
-1 \\
0 \\
0,198 \\
0\end{array}$ & $\begin{array}{l}T_{2}, y \\
T_{2}, y \\
-1 \\
-1 \\
1,288 \\
0,388\end{array}$ & $\begin{array}{l}T_{3}, y \\
T_{3}, y \\
-1 \\
-1 \\
-1,388 \\
1,388\end{array}$ & $\begin{array}{l}T_{4}, y \\
T_{3}, y \\
-1 \\
-1 \\
3,5 \\
2,388\end{array}$ & $\begin{array}{c}T_{5}, y \\
T_{5, y} \\
5 \\
0 \\
5 \\
0\end{array}$ & $\begin{array}{c}T_{5}, y \\
T_{5}, y \\
0 \\
0 \\
0 \\
0\end{array}$ \\
\hline 4 & $\begin{array}{l}f\left(0, T_{n}\right) \\
f\left(1, T_{n}\right)\end{array}$ & $\begin{array}{l}T_{1}, y \\
T_{5}, y\end{array}$ & $\begin{array}{l}T_{2}, y \\
T_{2}, y\end{array}$ & $\begin{array}{l}T_{3}, y \\
T_{3}, y\end{array}$ & $\begin{array}{l}T_{4}, y \\
T_{3}, y\end{array}$ & $\begin{array}{l}T_{5}, y \\
T_{5}, y\end{array}$ & $\begin{array}{l}T_{5}, y \\
T_{5}, y\end{array}$ \\
\hline
\end{tabular}
воряют этому уравнению.

Построение оптимальной стратегии для процесса фотолитографии

Как видно из таблицы, оптимальной для процесса фотолитографии является та стратегия, которая выбракует негодный объект на контрольных операциях $T_{0}$ и $T_{4}$, а с операции $T_{3}$ направит на исправление.

Заметим, что если финальный доход $h<4,779$, то оптимальная стратегия независимо от состояния объекта предпишет остановку процесса на контрольной операции $T_{0}$. Если же доход $h>6,014$, то оптимальная стратегия не допустит выбраковку объекта.

\section{ЛИТЕРАТ У РА}

1. G a rey, M. R., Bell Syst. Techn. J., 51, № 1, 291-300 (1972).

2. Hurst, E. G., Jr., Manag. Sci., 20, № 3, 378-384 (1973).

3. Т а мм ел а Ю. П., В кн.: Разработка автоматизированных систем управле:ия тех- 
нологическими процессами и автоматизированных систем испытания с обработкой данных при производстве силовых полупроводниковых приборов, Таллин, изд. НИИТЭ3, 1978 , с. $79-83 ; 84-89 ; 90-97$.

4. Britney, R. R., Manag. Sci., 18, № 9, 550-559 (1972).

5. Т а м мела Ю. П., В кн.: Разработка автоматизированных систем управления технологическими процессами и автоматизированных систем испытания с обработкой данных при производстве силовых полупроводниковых приборов, c. $97-102$.

6. Hin derer, K., Foundations of Non-Stationary Dynamic Programming with Discrete Time Parameter, Springer-Verlag, Berlin-Heidelberg-New York, 1970.

7. Д ынкин Е. Б., Юшкевич А. А., Управляемые марковские процессы и их приложения, М., «Наука», 1975.

8. Х в в р д Р. А., Динамическое программирование и марковские процессы, М., «Сов. радио», 1964.

9. М а й н Х., О с а к и С., Марковские процессы принятия решений, М., «Наука», 1977.

Научно-исследовательский и проектно-

технологический институт Таллинского электротехнического завода им. М. Н. Калинина
Поступнла в редакцию $24 /$ IX 1979

\section{J. TAMMELA}

\section{KEERUKA STRUKTUURIGA TOOTMISPROTSESSIDE OPTIMAALJUHTIMINE. TÄIELIKU INFORMATSIOONIGA MUDEL}

Stohhastilise tehnoloogilise liini juhtimise ülesanne on üldistatud juhule, kus protsessi tehnoloogilises skeemis esinevad kontuurid, s. o. töödeldav objekt võib pöörduda tagasi juba läbitud staadiumi. On näidatud juhitavate Markovi protsesside mudeli $\left[{ }^{6,7}\right]$ kasutamise vőimalikkust selliste protsesside kirjeldamisel ja optimeerimisel.

\section{J. TAMMELA}

\section{OPTIMAL CONTROL OF PRODUCTION PROCESSES WITH NON-SERIAL STRUCTURE. MODEL WITH COMPLETE INFORMATION}

The control problem of linear production process $\left[{ }^{1-5}\right]$ is generalized to the case with cycles. The processes in which the state of object under the process is observed in every stage, are studied.

A production process consists of the production operations and of the inspection stations. The structure of the process can be presented as an oriented graph $\left(T^{\prime}, \Delta^{\prime}\right)$, where $T^{\prime}$ is the set of the inspection stations and $\Delta^{\prime}$ is the set of the production operations.

Let us observe, after the $n$-th processing, $n=0,1,2, \ldots$ at inspection station $\tau_{n}$, the state of object $x_{n}$. After the observation, a certain decision must be chosen. Firstly, there exists a possibility of stopping the processing and, as a result of that, of receiving the final reward $h\left(x_{n}, \tau_{n}\right)$. Secondly, if there exists the operation $t_{n+1}=$ $=\left(\tau_{n}, \tau_{n+1}\right)$ in the process (i. e. if set $\left\{t: t=\left(\tau_{n}, \tau\right), t \in \Delta^{\prime}\right\}$ is a non-empty set), the processing can be continued and the production cost will increase by $c\left(x_{n}, t_{n+1}, y_{n+1}\right)$ if the $y_{n+1}$-type of processing takes place at the operation $t_{n+1}$. The processing changes the state of object according to the model of operations $p^{\prime}\left(\cdot \mid x_{n}, t_{n+1}, y_{n+1}\right)$, which is the conditional distribution for a new state of object. Thus the decision we chose must be the one which maximizes the expectation of the difference between the final reward and the production cost.

To be more exact, the stochastic dynamic decision problem $\left.{ }^{[6,7}\right]$ with the model $(S, A, D, p, r)$ defined by formulae (1)-(5), must be solved. If the decision model $(S, A, D, p, r)$ is finite, i. e. if both the state space $S$ and the space of actions $A$ are finite sets, the problem can be solved by Howard's iterative algorithm $\left[{ }^{8,9}\right]$. The photolitographic process which is presented as an example, is described by the finite model, and the optimal strategy of its control is found by the iterations presented in the table. 